\title{
Toe Tissue Transfer for Reconstruction of Damaged Digits due to Electrical Burns
}

\author{
Hyung-Do Kim, So-Min Hwang, Kwang-Ryeol Lim, Yong-Hui Jung, Sung-Min Ahn, \\ Jennifer K. Song \\ Department of Plastic and Reconstructive Surgery, Good Moonhwa Hospital, Busan, Korea
}

Background Electrical burns are one of the most devastating types of injuries, and can be characterized by the conduction of electric current through the deeper soft tissue such as vessels, nerves, muscles, and bones. For that reason, the extent of an electric burn is very frequently underestimated on initial impression.

Methods From July 1999 to June 2006, we performed 15 cases of toe tissue transfer for the reconstruction of finger defects caused by electrical burns. We performed preoperative range of motion exercise, early excision, and coverage of the digital defect with toe tissue transfer.

Results We obtained satisfactory results in both functional and aesthetic aspects in all 15 cases without specific complications. Static two-point discrimination results in the transferred toe cases ranged from 8 to $11 \mathrm{~mm}$, with an average of $9.5 \mathrm{~mm}$. The mean range of motion of the transferred toe was $20^{\circ}$ to $36^{\circ}$ in the distal interphalangeal joint, $16^{\circ}$ to $45^{\circ}$ in the proximal interphalangeal joint, and $15^{\circ}$ to $35^{\circ}$ in the metacarpophalangeal joint. All of the patients were relatively satisfied with the function and appearance of their new digits.

Conclusions The strategic management of electrical injury to the hands can be both challenging and complex. Because the optimal surgical method is free tissue transfer, maintenance of vascular integrity among various physiological changes works as a determining factor for the postoperative outcome following the reconstruction.

Keywords Burns / Electricity / Toes / Transfer
Correspondence: So-Min Hwang

Department of Plastic and

Reconstructive Surgery, Good

Moonhwa Hospital,

119 Beomil-ro, Dong-gu, Busan, Korea

Tel: +82-51-630-0199

Fax: +82-51-630-0145

E-mail: dowithdo@naver.com

Received: 1 Oct 2011 • Revised: 6 Feb 2012 • Accepted: 6 Feb 2012

pISSN: 2234-6163 • elSSN: 2234-6171 • http://dx.doi.org/10.5999/aps.2012.39.2.138• Arch Plast Surg 2012;39:138-142

\section{INTRODUCTION}

Due to the continued spread of the use of electricity as more countries industrialize, electrical burns are becoming a serious medical problem worldwide. Electrical burns are characterized as complex forms of crush and thermal injuries that result in complicated and serious damage to body parts such as the skin, vessels, nerves, muscles, and bones.

Hands are the most frequently affected sites of electrical burns. Hands burned by electricity show various pathological changes caused by multiple factors. Among those changes, the extent of vascular integrity is a determining factor for the results of reconstruction of hand parts burned by electricity, which are in many cases accompanied by digital defects and require free tissue transfer.

Since the first case of transferring a great toe to replace an amputated thumb was reported by Cobbet [1] in 1969, free great toe tissue transfer has been a reliable method to recover the mobility of injured hands. However, some patients refuse this procedure because they do not want to sacrifice their great toes 
for cosmetic or cultural reasons. For these patients, second toe tissue transfer has been used for thumb reconstruction. The authors found free tissue transfer from toes to be a useful method for reconstructing digital defects because it can make functional and cosmetic recovery possible and minimize the defects of donor sites.

\section{METHODS}

From July 1997 to June 2006, 15 cases of toe tissue transfer surgery were performed for the reconstruction of finger defects caused by electrical burns.

In the course of the treatment, the authors performed the appropriate mobility exercises for the joints and covered the digital defects using toe tissue transfer immediately after early resection of the burned sites, while recovering and reconstruction of the digital defects were performed as early as possible. The specific applied reconstruction methods were second toe transfer in 7 cases, first web space transfer in 4 cases, vascularized joint transfer in 2 cases, and toe pulp flap in 2 cases. All the patients were male with an average age of 24.6 years, ranging from age 3 to 38 (Table 1).

If the defect occurred only in soft tissue, the authors used first web space transfer or a toe pulp flap to reconstruct the tissue defects. When ankylosis occurred postoperatively at the proximal interphalangeal or metacarpophalangeal joint level, vascularized joint transfer was used for reconstruction and if the amputation occurred at the level of the metacarpophalangeal joint, we preferred second toe transfer to reconstruct the thumb defects.

The blood vessels were checked using a Doppler examination along with general preoperative tests. It has been reported that angiography is a safe method for measuring the extent of recipient blood vessel damage, but preoperative angiography had not been conducted except for unreliable Doppler views and wideranging damage.

Six of our patients underwent toe tissue transfer during the acute phase after the time of their injury ( 12 days on average after injury), and 9 patients underwent toe tissue transfer secondly (2.5 months on average after resurfacing the soft tissue defect).

In the dissection of portions of the hand, arteries, veins, and nerves, had been dissected along with the tissue requiring reconstruction at the damaged and defected sites, while in the dissection of portions of the foot, the greater saphenous vein, first dorsal metatarsal artery, and peroneal nerve had been dissected basically by creating a dorsal flap at the base of the second toe and the tissue necessary for reconstruction had been also dissected as appropriate for each case. Identifying the anatomical structure of the first dorsal metatarsal artery and dissecting it is considered one of the most important steps in toe tissue transfer.

After operative treatment, the patients started appropriate active or passive joint mobility exercises for their joints as early as possible. They were administered intravenous a mixture of antibiotics, dextran (500 IU/day) and heparin $(5 \mathrm{IU} / \mathrm{kg} / 8$ hours) intravenously during surgery and had the same volumes administered intravenously for 5 to 7 days continuously even after operation.

\section{RESULTS}

We analyzed 15 cases of toe tissue transfer for reconstructing finger defects caused by electrical burns. According to Gilbert's

\section{Table 1. Summary of 15 patients}

\begin{tabular}{|c|c|c|c|c|c|c|}
\hline Case & Sex/Age & Current & Defect site & Donor & FDMA type & Follow-up (mo) \\
\hline 1 & $M / 36$ & High & Thumb, Rt. & 2nd toe, Lt. & ॥ & 36 \\
\hline 2 & $\mathrm{M} / 38$ & High & Index, Rt. & 2nd toe, Rt. & la & 28 \\
\hline 3 & $M / 7$ & Low & Ring, Lt. & 1st web, Rt. & $\mathrm{lb}$ & 26 \\
\hline 4 & $\mathrm{M} / 22$ & Low & Little, Rt. & 2nd PIP JT, Lt. & III & 24 \\
\hline 5 & $\mathrm{M} / 36$ & High & Thumb, Rt. & 2nd toe, Lt. & $\mathrm{lb}$ & 32 \\
\hline 6 & $\mathrm{M} / 3$ & Low & Index, Lt & 2nd pulp, Lt. & $\mathrm{lb}$ & 24 \\
\hline 7 & $\mathrm{M} / 26$ & High & Thumb, Lt. & 1st web, Lt. & $\mathrm{lb}$ & 15 \\
\hline 8 & $\mathrm{M} / 16$ & High & Index, Lt & 2nd toe, Lt. & la & 20 \\
\hline 9 & $\mathrm{M} / 28$ & High & Thumb, Rt. & 2nd toe, Lt. & III & 21 \\
\hline 10 & $\mathrm{M} / 16$ & Low & Index, Lt & 2nd MP JT, Lt. & ॥ & 18 \\
\hline 11 & $\mathrm{M} / 29$ & High & Little, Rt. & 2nd toe, Lt. & II & 15 \\
\hline 12 & $\mathrm{M} / 24$ & High & Thumb, Rt. & 1st web, Lt. & la & 14 \\
\hline 13 & $\mathrm{M} / 28$ & High & Index, Lt & 1st pulp, Lt. & la & 15 \\
\hline 14 & $\mathrm{M} / 24$ & High & 1st web, Rt. & 1st web, Lt. & ॥ & 12 \\
\hline 15 & $\mathrm{M} / 22$ & High & Thumb, Rt. & 2nd toe, Lt. & $\mathrm{lb}$ & 8 \\
\hline
\end{tabular}


classification, the form of the first dorsal metatarsal artery was classified into type $1 \mathrm{~A}$ in 4 cases, type $1 \mathrm{~B}$ in 5 cases, type 2 in 4 cases, and type 3 in 2 cases.

The follow-up observation extended for 8 to 36 months with a 23.1 month average. It was confirmed that all of the transferred flaps were survived, and the total operating time was 5 to 8 hours, with an average of 6.5 hours.

In all of the patients, sensory recovery of the reconstructed digits was estimated by static two-point discrimination (S-2PD) excluding the vascularized joint transfer. The S-2PD results in the transferred toe ranged from 8 to $11 \mathrm{~mm}$, with an average of $9.5 \mathrm{~mm}$. The mean range of motion of the reconstructed digits was $20^{\circ}$ to $36^{\circ}$ in the distal interphalangeal joint, $16^{\circ}$ to $45^{\circ}$ in the proximal interphalangeal joint, and $15^{\circ}$ to $35^{\circ}$ in the metacarpophalangeal joint. All the patients were relatively satisfied with the function and appearance of their new digits, and thus from both functional and cosmetic perspectives, satisfactory results had been obtained without any specific complications in any of the 15 cases.

\section{Case 1}

A 36-year-old man who had lost his right thumb at the metacarpophalangeal joint by high-voltage electrical burns was initially covered by adipofascial forearm turn over flap and reconstructed using a transfer of the second toe to the thumb later on, and two years after the second toe transfer for thumb reconstruction, and $13 \mathrm{~mm}$ of S-2PD had been restored in his right hand (Fig. 1).

\section{Case 2}

A 7-year-old boy with necrotic change of soft tissue at the left middle, ring, and little fingers after low-voltage electrical burns had a tendon and bone exposed after debridement at his left ring finger, which was covered by a first web space flap with the postoperative outcome at 15 months showing a satisfying result (Fig. 2).

\section{Case 3}

A 22-year-old man who had stiffness of the proximal interphalangeal joint at the right little finger by electrical burns, underwent reconstruction using vascularized joint transfer, and 15 months after surgery, the stiff joint can be flexed more than $40^{\circ}$. The patient reported satisfaction with the results (Fig. 3).

\section{DISCUSSION}

The clinical patterns of electrical burns are complex and diverse, with multiple variables determining the extent and range of elec-

\section{Fig. 1. Second toe transfer (case 1 in table 1)}

(A) Loss of Right thumb at the metacarpophalangeal joint by hightension electrical burns. (B) Two years after second toe transfer for thumb reconstruction. Pinch (60\%), opposition, and two point discrimination (13 $\mathrm{mm}$ ) has been obtained. (C) Postoperative donorsite appearance after 6 months.
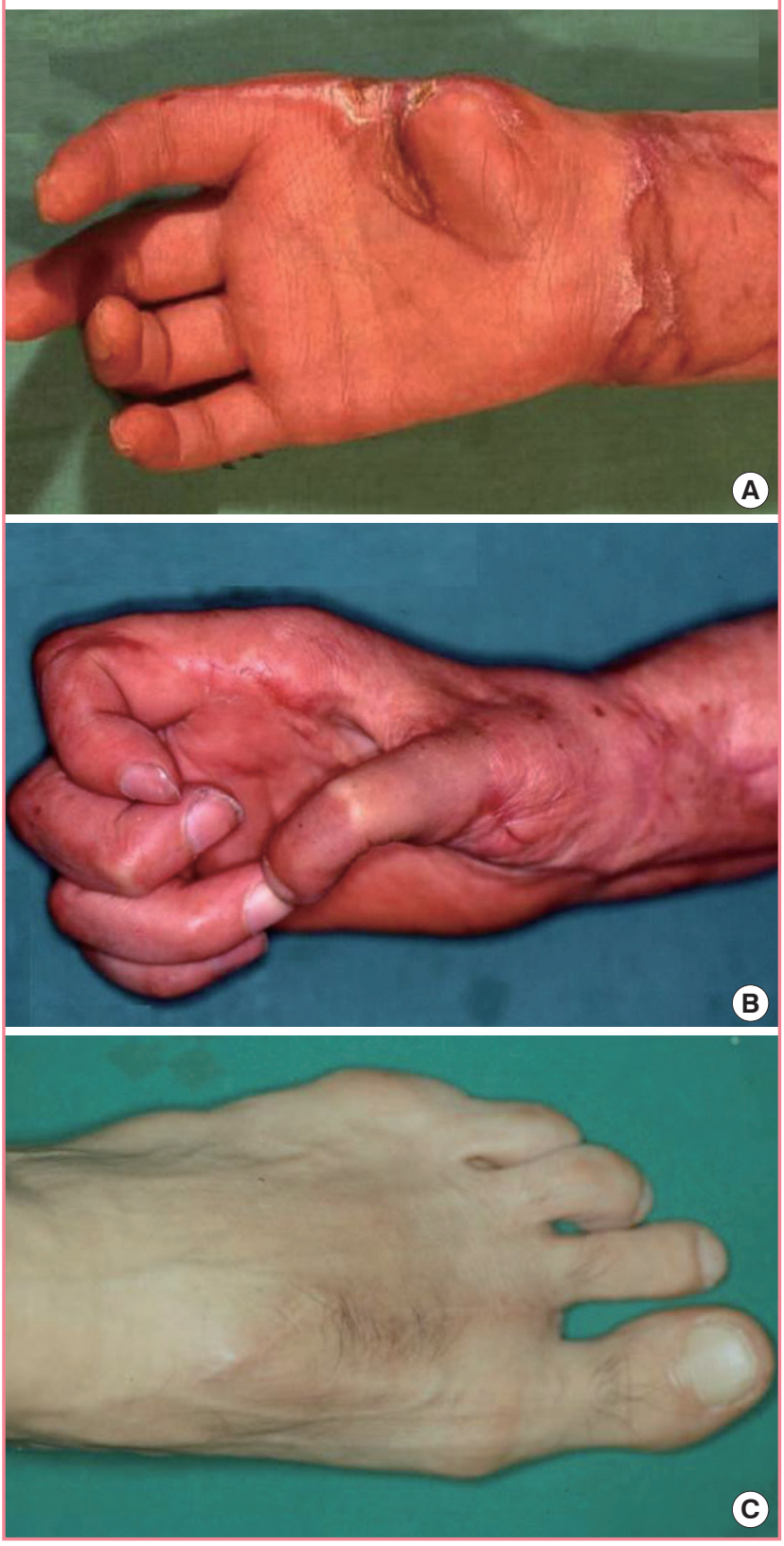

trical burns; these variables such as the voltage, electrical current volume, tissue resistance, type of electrical current, body part contacted by electricity, and the size of the affected area, react mutually with each other.

However, as electrical burns are usually classified as high-voltage electrical burns or low-voltage electrical burns, high-voltage electrical burns are caused by a current of more than 1,000 volts, and low-voltage electrical burns are caused by a current of less 


\section{Fig. 2. First web space flap (case 3 in table 1)}

(A) Necrotic change of soft tissue at left middle, ring, little finger by low-tension electrical burns. Tendon and bone was exposed after debridement at ring finger. (B) First web space flap is designed from right foot. (C) Postoperative outcome after 15 months.
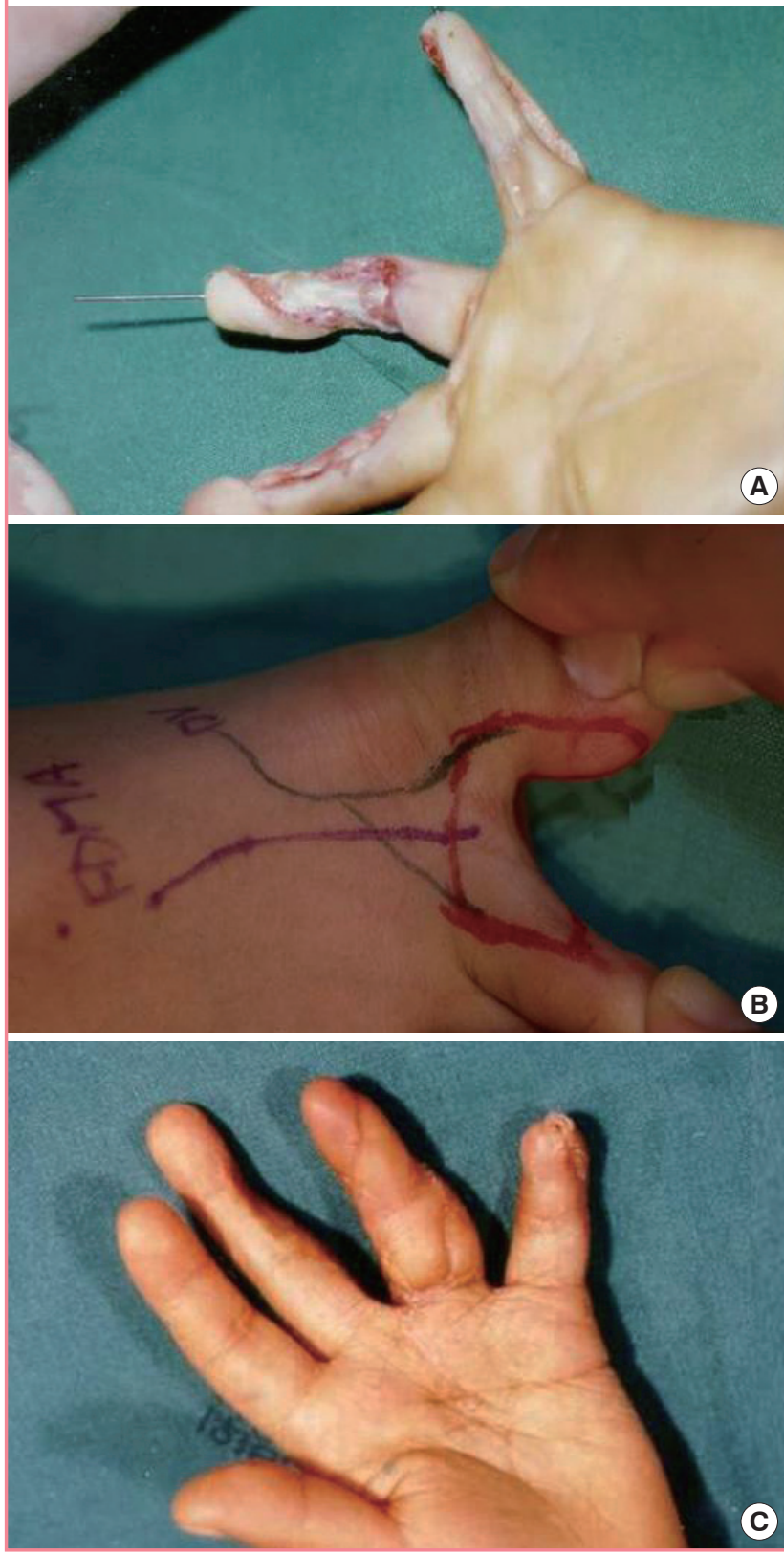

than 1,000 volts. In our cases, the highest voltage was 22,000 volts and the lowest voltage was 220 volts.

In electrical burns, tissue damage lapse is addressed by the theory of progressive damage or the theory of non-progressive damage. According to progressive damage theory, heat produced by the passage of an electric current is in direct proportion to the amount of tissue resistance and the blockade of the artery that finally results in tissue death [2].

\section{Fig. 3. Vascularized joint transfer (case 4 in table 1)}

(A) Stiffness of proximal interphalangeal joint (PIP) joint at little finger by electrical flame burns. (B) PIP joint flap of second toe is elevated on its vascular pedicle. (C) Fifteen months after surgery. Stiffed joint is flexed more than $40^{\circ}$
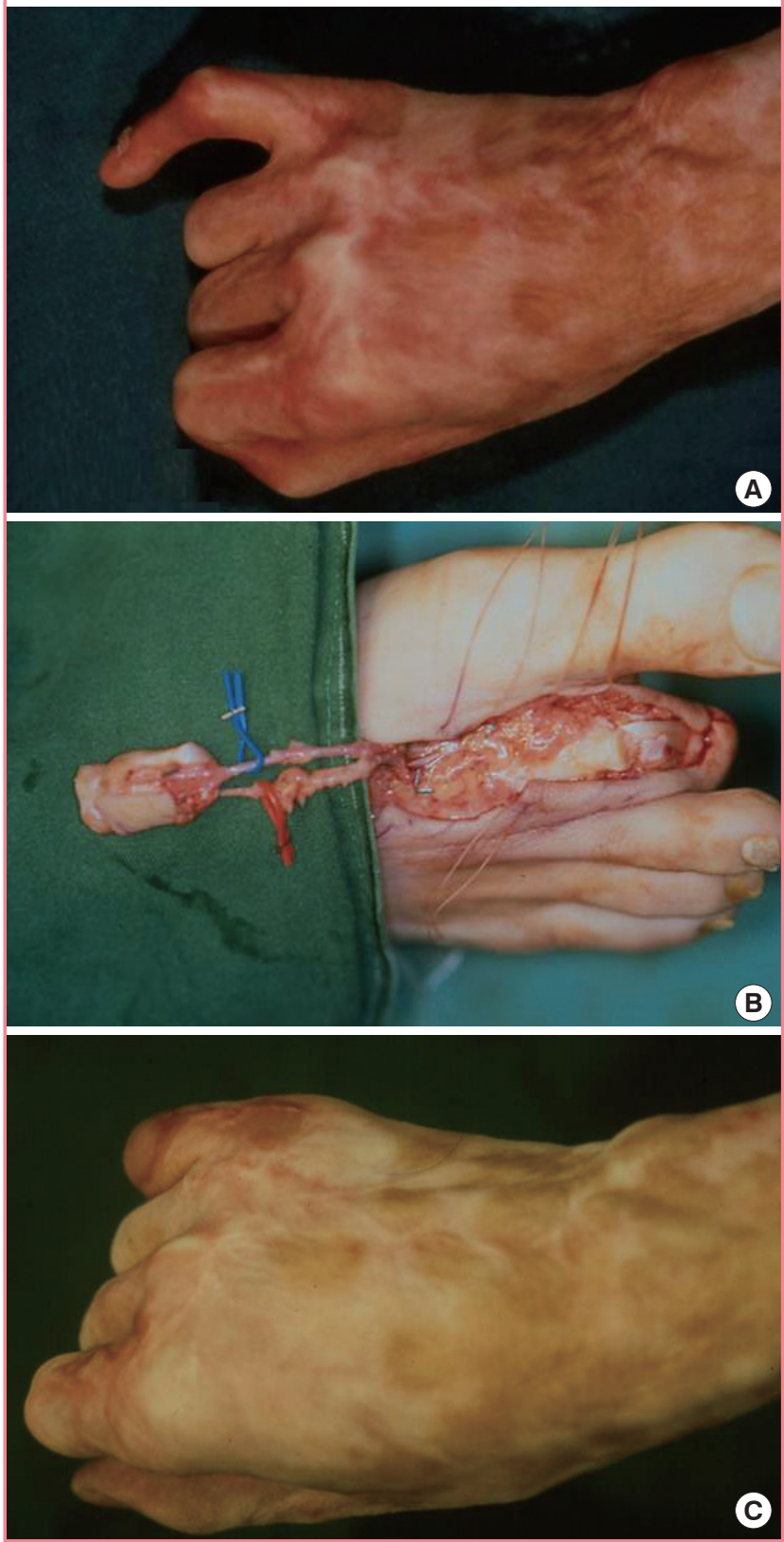

However, according to non-progressive damage theory, destruction of soft tissue is caused by problems of minute blood circulation or a blood clot that can be triggered by a secondary infection or the heat produced by the electric current at the time of injury [3-5].

It is possible to predict the level of ultimate amputation of the limbs by the results of a minute blood vessel scan, which should be conducted right after a patient's limbs are burned, but 
if ongoing death of limb tissue occurs, it means there are other factors in addition to problems with the blood vessels [5].

The theory of non-progressive damage has recently been accepted by most surgeons, and according to the theory, it is recommended that the burned area be reconstructed as early as possible to preserve the function of damaged parts, rehabilitate the patient as early as possible, and reduce the duration of morbidity and hospitalization. The procedure fulfills the primary goal of treating electrical burns, which is for patients to recover excellent function, cosmetic form, and to restore the ability to perform normal daily activities [6].

It was first reported by Hagan et al. [7] in 1982 that the free flap had been used for acute electrical burns. Deep electrical burns on the legs were cured by the use of the latissimus dorsi musculocutaneous free flap and split-thickness skin grafts. In 1969, Cobbett [1] performed the first successful microvascular great toe transfer to replace an amputated thumb, which has been performed since then by hand surgeons worldwide to replace missing thumbs.

High-voltage electrical burns usually cause serious defects and are not expected to recover a satisfactory function even if a great toe transfer is conducted. We performed second toe transfer for thumb reconstruction instead of using great toe transfer because, second toe transfer can be a good alternative for patients who do not want to sacrifice their great toes for cosmetic or cultural reasons and want fewer donor defects.

The frequency of using free tissue transfer for curing obstinate burn wounds is currently increasing and vessel injury is becoming a problem in electrical burn injuries, as up to several centimeters proximal to the body is usually involved in the injury [8]. With this important point in mind at the time of free flap, the dissection of the vessel should be performed carefully and safely during surgery. The proper digital artery and superficial branch of the radial artery have usually been used as the recipient artery, and as mentioned earlier, the recipient artery was confirmed by using a Doppler study, and angiography was conducted for confirmation in the severe cases. In the case of digital soft tissue defects caused after injury, surgery was conducted as early as possible during the acute phase (12 days on average after injury) due to advantages such as decreasing chances of infection, seeking early functional recovery, and allowing easy dissection of the intact neurovascular pedicle through the open wound. In the case of a large soft tissue defect and reconstruction of amputated digits, surgery was performed (2.5 months on average after resurfacing the soft tissue defect) after proceeding with soft tissue coverage of the amputated stump. Complete flap survival was observed in all cases. Three flaps suffered from venous congestion, but they were not re-explored because they showed a stable, non-progressive blue discoloration.

The authors consider free tissue transfer from toes to be a useful method for reconstructing digital defects because it makes possible the recovery of functionality and cosmesis, and also minimizes donor site defects. The existing techniques of toe tissue transfer have largely satisfied the requirements of restoring function through digital defect reconstruction. However, the final aesthetic outcome is often disappointing to the patients. We were able to obtain a satisfactory aesthetic outcome by designing the flap to fit the wound and obtaining satisfactory functional restoration using toe tissue transfer.

The hand is the area most commonly affected by electrical burn injuries due to the accessibility of electricity, and this type of injury represents a distinct medical condition in which functional and aesthetic sequelae are very important. This makes the strategic management of electrical injuries on hands both challenging and complex. Because the optimal surgical method is free tissue transfer, maintenance of vascular integrity among various pathologic changes works as a determinate factor for the postoperative outcome following reconstruction.

\section{REFERENCES}

1. Cobbett JR. Free digital transfer. Report of a case of transfer of a great toe to replace an amputated thumb. J Bone Joint Surg Br 1969;51:677-9.

2. Robson MC, Murphy RC, Heggers JP. A new explanation for the progressive tissue loss in electrical injuries. Plast Reconstr Surg 1984;73:431-7.

3. Mann R, Gibran N, Engrav L, et al. Is immediate decompression of high voltage electrical injuries to the upper extremity always necessary? J Trauma 1996;40:584-7.

4. Chick LR, Lister GD, Sowder L. Early free-flap coverage of electrical and thermal burns. Plast Reconstr Surg 1992;89:1013-9.

5. Buchanan DL, Erk Y, Spira M. Electric current arterial injury: a laboratory model. Plast Reconstr Surg 1983;72:199-207.

6. Sturim HS. The treatment of electrical injuries. J Trauma 1971;11:959-65.

7. Hagan KF, Buncke HJ, Gonzalez R. Free latissimus dorsi muscle flap coverage of an electrical burn of the lower extremity. Plast Reconstr Surg 1982;69:125-9.

8. Peterson RA. Electrical burns of the hand. Treatment by early excision.J Bone Joint Surg Am 1966;48:407-24. 